\title{
News on Modeling of Walking Robot Critical Positions
}

\author{
Marcel MIGDALOVICI ${ }^{1}$, Luige VLADAREANU* ${ }^{1}$, Nicolae POP $^{1}$, Victor VLADAREANU ${ }^{1}$, \\ Alexandru VLADEANU ${ }^{2}$, Daniela BARAN ${ }^{3}$, Gabriela VLADEANU ${ }^{1}$ \\ ${ }^{1}$ Romanian Academy, Institute of Solid Mechanics of the Romanian Academy, Bucharest \\ ${ }^{2}$ University of Civil Engineering, Bucharest, \\ ${ }^{3}$ National Institute of Aerospace Research “Elie Carafoli”, Bucharest.
}

\section{Article Info}

Received Nov $3^{\text {rd }}, 2018$

\section{Keyword:}

environment's model axiom, walking robot, kinematics / dynamic model, stability regions, axiomatic system.

\begin{abstract}
The principal objective of our study is to emphasize the strategies for the walking robot mathematical model to traverse an uneven terrain, respecting the hypothesis of environment model defined by us. The multiple aspects on axiomatic systems, with possible application to environment's mathematical model axiomatization, open an interesting new way of research and is exposed in the first part of the paper. Our study on the walking robot begins with formulation of improved mathematical model for physical identification derived from geometrical identification of critical position in particular case of waking robot leg. The physical identification of the critical position is analyzed in the paper on our concrete case of walking robot leg mathematical model. The specialized algorithm performed by us is used for verification of the theory. The new directions of research, opened by our analyses in this area, are described.
\end{abstract}

\section{Corresponding Author:}

Luige Vladareanu,

Romanian Academy, Institute of Solid Mechanics, 15 Constantin Mille street, 010141 Bucharest, Romania.

Email: luige.vladareanu@vipro.edu.ro

\section{INTRODUCTION}

The walking robot models are assumed to respect the mathematical modeling of the environment where each robot evolution is performed. The first part of the exposure is dedicated to axiomatic environment model. The mathematical describing of the environment is realized through the kinematics or dynamic system models in the general case when system depends on parameters. The system models are defined using relevant parameters as physical parameters, geometrical parameters, possible chemical, biological, economical, etc, as shown in Migdalovici et al.(2015), Vladareanu et al.(2009), Vladareanu et al.(2013),Vladareanu et al.(2015), Pop et al.(2014), Dinca et al.(2004), Migdalovici et al.(2010), Hirsch et al.(2004), Guckenheimer and Holmes (1984), Halanay and Rasvan (1993), Voinea and Stroe (2000), Hayashi (1964), Feng ( 2016), Staretu (2015) and Wang (2015).

The important property observed by us on the dynamic system models that approach the phenomenon from the reality is property of separation between stable and unstable regions from the free parameters domain of 
the system. This property is assumed as one characteristic of environment's mathematical model. Mathematical hypothesis on the kinematics or dynamic system matrix components, in the linear case, that assure separation between existence and inexistence regions from the free parameters domain in kinematics case or between stable and unstable regions from the free parameters domain in the dynamic case are discussed.

Our walking robot leg kinematics model and corresponding dynamic model with application on selected case of walking robot leg, respecting environment model, are detailed in the second part of the exposure.

Possible critical positions of the walking robot in its evolution are identified using an inverse method proposed by us. The link between dynamic model and corresponding kinematics model as limit case of dynamic model of walking robot is commented. Aspect referred to problem studied using kinematics model that can be studied and in the analog dynamic model having a similar solution, is also analyzed.

Many researches associated with our studies on axiomatic environment's model are produced. The book of Judea Pearl (2009) on Causality, using axiomatic characterization of one model, may be of environment model, is firstly mentioned. Another book entitled "The Incomplete Universe: Totality, Knowledge, and Truth" by Patrick Grim (1991) is also in area of interest referred to our research.

\section{ON DEFINITION OF ENVIRONMENT'S MATHEMATICAL MODEL}

The mathematical property identified by us on all dynamic systems that approximate phenomena from the reality, in general case of systems that depend on parameters, is property of separation between stable and unstable regions from the free parameters domain of the system.

We remark, for the first in literature, mathematical conditions on the functions that define the linear or nonlinear dynamic system, which assure the separation between stable and unstable regions in the free parameters domain of the system.

The real matrix that intervenes in definition of the linear dynamic system or in definition of linear "first approximation" of the nonlinear dynamic system in general case of system that depends on parameters, is denoted by $A$ and assumed as matrix from $R^{n \times n}, n \in N$. Below we discuss on $\mathrm{QR}$ algorithm for Hessenberg form of the real matrix $A$.

The matrix $A \in R^{n \times n}$ is assumed with real elements $a_{i j}, i=1, \ldots, n ; j=1, \ldots, n$., and in Hessenberg form such that its elements $a_{i j}=0$ for $2<i \leq n, j<i-1$ and also is firstly assumed with distinct eigenvalues, real or complex. Any real matrix $A$ can be associated with a similar matrix in Hessenberg form, and substituted by it for stability analysis, because they have the same eigenvalues, essential condition for stability identical comportment.

The QR algorithm is applied on the matrix $A$ in Hessenberg form and for each complex eigenvalues $\alpha \pm i \beta$, if there exists, appear corresponding real matrix of two order $\left[\begin{array}{cc}\alpha & \beta \\ -\beta & \alpha\end{array}\right]$ on the diagonal of the real final equivalent Schur form of the matrix $A$ and for each distinct real eigenvalue appear also on the diagonal of the Schur form of the matrix $A$. Some aspects on Schur form of the matrix $A$ are related by Francis (1962), Wilkinson (1965) and Parlet (1968). The QR algorithm referred to convergence of matrix $A$ to Schur form of the matrix, for real matrix $A$ in Hessenberg form, is studied by Wilkinson (1965) and Parlet (1968).

The matrix of the form $A-\lambda I$, deduced from matrix $A$ in Hessenberg form, having real or complex value $\lambda$, is also in Hessenberg form and is denoted "shift of origin" for the matrix $A$. The "shift of origin" method assures transposition of real matrix in complex domain through suitable complex value $\lambda$ that contributes to simplify stability analysis of the dynamic system defined by one matrix.

The QR algorithm by shift of origin for the matrix $A$ is related by Parlet (1968) through relations:

$$
\begin{aligned}
& Q_{s}\left(A_{s}-k_{s} I\right)=R_{s}, \\
& A_{s+1}=R_{s} Q_{s}^{T}+k_{s} I=Q_{s} A_{s} Q_{s}^{T}, s=1,2, \ldots
\end{aligned}
$$

The initial matrix $A$ of the system is denoted in the QR algorithm by $A_{1}$ and assumed in Hessenberg form, 
$Q_{s}$ is orthogonal matrix, $A_{s}, s \geq 2$ is in Hessenberg form, $k_{s}$ is "shift of origin", and matrix $R_{s}$ is in upper triangular form. The matrices $A_{s}, A_{s+1}, s=1,2, \ldots$ are similar. The shift of origin, having initial value $\lambda$ sufficient close to selected matrix eigenvalue, real or complex, assures acceleration of the $\mathrm{QR}$ algorithm convergence to the similar diagonal form of the matrix. This is an important motivation to use QR algorithm by shift of origin.

The above analysis is performed in hypothesis that eigenvalues of real matrix are different values. For analysis in the case of real matrix multiple eigenvalues, we call the results from matrix theory mentioned by Hirsch et al. (2004), which verified on the linear normed space $L\left(R^{n}\right)$ of matrices that the set of matrices with distinct eigenvalues from the space $L\left(R^{n}\right)$ is an open and dense set in space $L\left(R^{n}\right)$.

The above property assures the possibility to identify some qualities, which arise from stability analysis on linear dynamic system defined by real matrices with distinct eigenvalues to close dynamic system defined by matrix with multiple eigenvalues. In the following we describe our conditions on functions that define the autonomous linear dynamic system or nonlinear dynamic system "first approximation" that assure the separation of stable and unstable regions in parameters domain. Our result is formulated as:

Theorem on separation: If autonomous linear dynamic system defined by matrix $A$, has continuous on piecewise components of the matrix as functions of dynamic system free parameters and is assured that eigenvalue functions of the matrix $A$ are also continuous on piecewise then these conditions permit separation between stable and unstable regions of the dynamic system in parameters domain. Such property permits a mathematical characterization of the environment and is proposed by us as an axiom of environment mathematical model.

The book of Patrick Grim (1991) on Incomplete Universe, calling Gödel results on incompleteness phenomena referred to formal systems, is mentioned and suggests possible new ways of researches.

Remark: The property of separation in the domain of free parameters is referred on existence and inexistence regions of parameters, in case of kinematics model studied in general case of system that depends on parameters. Such case is analyzed through our application on walking robot leg model, which respects environment model.

\section{ANALYSIS ON PARTICULAR CASE OF ENVIRONMENT'S SYSTEM}

Our particular model of walking robot leg, firstly analyzed in kinematics case, having physical model of robot leg (figure. 1) described by a "pivot point" $\mathrm{O}_{\mathrm{c}}$ (assumed fixed in this analysis) jointed to robot body and by two bars $\mathrm{O}_{\mathrm{c}} \mathrm{P}$ and $\mathrm{PQ}$ jointed also in point $\mathrm{P}$ (see as example Migdalovici et al.(2015)).

The point $P$ named "knee joint" of leg and point $Q$ named "base point" of leg, together displaced in same vertical plane, related in figure 1 . The case analyzed below on fixed pivot point is developed assuming point $\mathrm{P}$ that traverses a circle arc route with centre $\mathrm{O}_{\mathrm{c}}$ and assuming that base point $Q$ traverses a close route précised below.

The route of point $Q$ is compounded from the superior ellipse $\operatorname{arc} Q_{B} Q_{A}$ having the ellipse centre $O_{E}$ and the semiaxes $a, b$, and from closure of the point $\mathrm{Q}$ route in cycled evolution identified by horizontal segment $\mathrm{Q}_{\mathrm{A}} \mathrm{Q}_{\mathrm{B}}$ traversed by the base point $\mathrm{Q}$.

The points $P_{1}, P_{A}, P, P_{B}$ are knee joint positions in leg cyclic evolution, points $Q_{1}, Q_{A}, Q, Q_{B}$ are base point positions in leg cyclic evolution, and points $\mathrm{P}_{\mathrm{A}}^{*}, \mathrm{P}_{\mathrm{B}}^{*}, \mathrm{Q}_{\mathrm{A}}^{*}, \mathrm{Q}_{\mathrm{B}}^{*}$ are critical positions. The parameters assumed in our model and the orthogonal system of coordinates also assumed in the model are clarified from the coordinates values on the figure points: $\mathrm{O}_{\mathrm{c}}(a, h), \mathrm{O}_{\mathrm{E}}\left(a, b_{1}\right), \mathrm{P}\left(x_{P}, y_{P}\right), \mathrm{Q}\left(x_{Q}, y_{Q}\right), \mathrm{Q}_{\mathrm{A}}\left(x_{A}, 0\right), \mathrm{Q}_{\mathrm{B}}\left(x_{B}, 0\right)$. The parameters values are selected in following analysis such that physical model from figure 1 is possible. 
The points $P_{A}^{*}$ and $P_{B}^{*}$ from figure signify extremities of the domain on circle arc where knee joint $P$ is displaced without changing direction of displacement, such extremities points being geometrical identified by normality of segments $P_{A}^{*} Q_{A}^{*}$ and $P_{B}^{*} Q_{B}^{*}$ on ellipse arc.

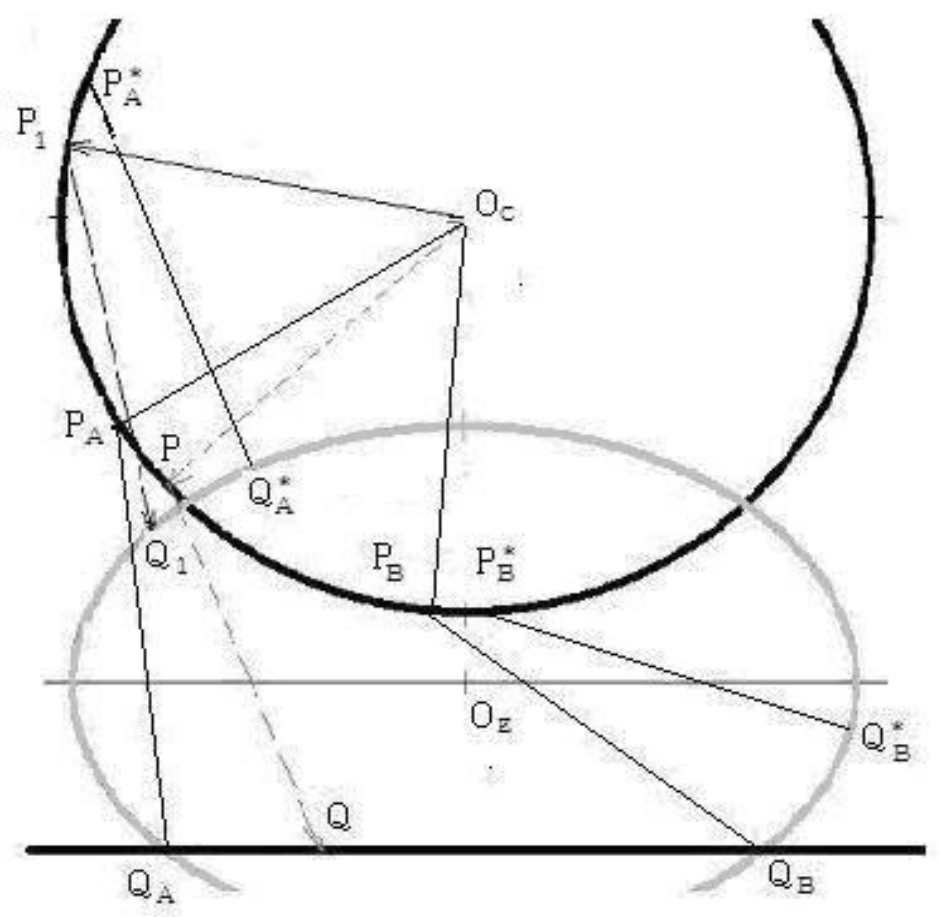

Figure 1: Physical model of the walking robot leg

The positions $P_{A}^{*}$ and $P_{B}^{*}$ of knee joint, described by us on particular case of figure 1, are named "critical points" in leg evolution. In other cases of robot leg with fixed pivot point, defined by the values of the geometrical parameters or in cases where pivot point is moved in walking robot evolution, is important to search as in case described here, possible existence of knee joint critical positions where direction of displacement is changed and where speed of knee joint must be zero for natural continuous evolution.

In real cases of walking robot is necessary to respect existence of critical positions because in these positions the speed of knee joint is of zero value imposed by changing of the displacement direction for the knee joint and ignorance of this aspect will block real evolution of the walking robot. The mathematical model that corresponds to physical model, selected by particular case represented in figure 1 , is initialized calling the equations:

$y_{\mathrm{P}}=h+\left(2 a x_{\mathrm{P}}-x_{\mathrm{P}}^{2}\right)^{1 / 2} ; y_{\mathrm{Q}}=(b / a)\left(2 a x_{\mathrm{Q}}-x_{\mathrm{Q}}^{2}\right)^{1 / 2}+b_{1} ;$

$x_{\mathrm{Q}}(t)=-v_{0} t+2 a / 3 ;\left(x_{\mathrm{P}}-x_{\mathrm{Q}}\right)^{2}+\left(y_{\mathrm{P}}-y_{\mathrm{Q}}\right)^{2}-a^{2}=0 ;$

The above equations are fulfilled by the coordinates of knee joint $\mathrm{P}$ and of base point $\mathrm{Q}$. The parameters' values of the model from figure 1 must respect the conditions $a>b>b_{1}>0 ; 2 a>h$, where $a$ and $b$ are ellipse's semiaxes. The variable $x$, in our mathematical model, is selected to have linear evolution between 0 and $2 a$, excepting a neighbourhood around each critical point, if it exists, in our case of fixed pivot point of the leg, assuming proper values for constant speed $v_{0}$ and initial condition $x_{0}$, as below:

$x(t)=v_{0} t+x_{0}$

One cycle on the robot leg evolution is assumed, according to our physical leg model, that starts from the 
position of base point $Q_{B}$, traversing the superior ellipse arc up to the point $Q_{A}$, using the evolution law (3), and returns by linear uniform displacement on horizontal axle, in the point $\mathrm{Q}_{B}$, excepting a neighbourhood around each position $\mathrm{Q}_{\mathrm{A}}^{*}, \mathrm{Q}_{\mathrm{B}}^{*}$, where an admissible evolution is selected.

The time parameter, for $x_{Q}(t)$ decreasing convergent to $x_{Q A^{*}}$ and the equation $\mathrm{d}\left(y_{P}(t)\right) / \mathrm{d} t=0$ fulfilled, taking into account admissible value for parameter $v_{0}$, is of our interest because this equation defines critical position, if it exists, of base point $\mathrm{Q}$ and knee joint $\mathrm{P}$. Another equation that helps us to identify the critical time corresponding to critical position is deduced by derivation of the last equation from relations (2) as function on time. The mathematical formulas that help us to perform the algorithm to identify the critical time are specified below.

$$
\begin{aligned}
& \operatorname{val}\left(x_{\mathrm{P}}(t)\right)=2 a x_{\mathrm{P}}(t)-\left(x_{\mathrm{P}}(t)\right)^{2} ; y_{\mathrm{P}}(t)=h+\operatorname{val}\left(x_{\mathrm{P}}(t)\right)^{1 / 2} ; \\
& \mathrm{d}\left(y_{\mathrm{P}}(t)\right) / \mathrm{d} t=\left(\mathrm{d}\left(y_{\mathrm{P}}\right) / \mathrm{d} x_{\mathrm{P}}\right)\left(\mathrm{d} x_{\mathrm{P}}(t) / \mathrm{d} t\right)=\left(\left(a-x_{\mathrm{P}}(t)\right) /\left(y_{\mathrm{P}}(t)-h\right)\right)\left(\mathrm{d} x_{\mathrm{P}}(t) / \mathrm{d} t\right) ; \\
& \operatorname{valy}\left(x_{\mathrm{P}}(t)\right)=\left(a-x_{\mathrm{P}}(t)\right) /\left(y_{\mathrm{P}}(t)-h\right) ; \mathrm{d}\left(y_{\mathrm{P}}(t)\right) / \mathrm{d} t=\operatorname{valy}\left(x_{\mathrm{P}}(t)\right)\left(\mathrm{d} x_{\mathrm{P}}(t) / \mathrm{d} t\right) ; \\
& \mathrm{d}\left(y_{\mathrm{P}}(t)\right) / \mathrm{d} t=\operatorname{valy}\left(x_{\mathrm{P}}(t)\right)\left(\mathrm{d} x_{\mathrm{P}}(t) / \mathrm{d} t\right) \\
& \left.\left(x_{\mathrm{P}}(t)-x_{\mathrm{Q}}(t)+\left(y_{\mathrm{P}}(t)-y_{\mathrm{Q}}(t)\right) \operatorname{valy}\left(x_{\mathrm{P}}(t)\right)\right)\left(\mathrm{d} x_{\mathrm{P}}(t) / \mathrm{d} t\right)\right)=f\left(x_{\mathrm{P}}(t), t\right)
\end{aligned}
$$

The function $f\left(x_{\mathrm{P}}(t), t\right)$ from (4) is a known function of $t$ and $x_{\mathrm{P}}(t)$. The equation $\mathrm{d}\left(y_{\mathrm{P}}(t)\right) / \mathrm{d} t=$ $=\operatorname{valy}\left(x_{\mathrm{P}}(t)\right)\left(\mathrm{d} x_{\mathrm{P}}(t) / \mathrm{d} t\right)$ from (4) helps us to define the critical time, if it exists. This way of leg critical position identification is distinct of our geometrical identification initially described and this last method is named by us as "physical identification" of critical position. The algorithm for physical identification of critical time in our particular model consists in solving of equation in time $f\left(x_{P}(t), t\right)=0$ deduced from (4) by specification of compatible selected free parameters. The method applied in this particular case can inspire us to extend identification of possible critical positions for more complicated models.

The domain of existence, in these formulated cases of kinematics analysis, coincides with the domain of stability for the walking robot leg model having negligible mass such that we remark that can intuitively affirm existence of a separation between stable and unstable regions in the free parameters domain of the robot leg dynamic model assuming uniform distributed mass on the leg components.

The complex problems that appear in the robotics area and not only, which we are interested in our study on walking robots, are also described in the book of Stuart J. Russell and Peter Norvig (2016) on artificial intelligence as modern approach.

\section{CONCLUSIONS}

The environment mathematical characterization is opened by firstly proposed axiom verified on all dynamical systems from the literature. The next objective of our research on walking robot critical position is to perform an algorithm for physical identification of critical position on trajectory done and to apply the virtual result on real case. The environment's mathematical axiomatization and walking robot critical positions are not exhausted by our study but an interesting way of research is initialized. The classical law that "one system of axioms sufficient expressive is incomplete" represent for our research about environment's model axiomatization an inspiration argument for further directions of study.

\section{ACKNOWLEDGEMENTS}

This work was supported by a grant of the Romanian Ministry of Research and Innovation, CCCDIUEFISCDI, MULTIMOND2 project number PN-III-P1-1.2-PCCDI2017-0637/33PCCDI/01.03.2018, and by KEYTHROB project, number PN-III-P3-3.1-PM-RO-CN-2018-0144 / 2 BM/2018, within PNCDI III, and by the European Commission Marie Skłodowska-Curie SMOOTH project, Smart Robots for Fire-Fighting, H2020-MSCA-RISE-2016-734875. The authors gratefully acknowledge the support of the Robotics and Mechatronics Department, Insitute of Solid Mechanics of the Romanian Academy. 


\section{REFERENCES}

[1] Dinca F. Zaharia E. Baran D. An analysis of chaotic evolutions of dynamic systems, Revue Roumaine des science techniques, 49, pp. 75-90, (2004).

[2] Feng Yongfei, Wang Hongbo, Lu Tingting, Vladareanu Victor, Li Qi, Zhao Chaosheng.(2016), Teaching Training Method of a Lower Limb Rehabilitation Robot, International Journal of Advanced Robotic Systems, 13(57): 1-11.

[3] Francis J.G.F. QR transformation, The Computer Journal, 4, First part, pp. 265-271, (1961), Second part, pp. 332-345, (1962), https://doi.org/ 10.1093/comjn1/4.4.332.

[4] Grim P. The Incomplete Universe: Totality, Knowledge, and Truth, The MIT Press. (1991).

[5] Stuart J.R. and Peter N. Artificial Intelligence: A modern Approach, Pearson Education Limited, England, (2016).

[6] Guckenheimer J. Holmes P. Nonlinear Oscillation Dynamical Systems and Bifurcation of Vector Fields, Springer Verlag, Berlin, Heidelberg, Tokyo, (1984).

[7] Hayashi, C. Nonlinear Oscillations in Physical Systems, Ed. McGraw - Hill Book Company, New York, San Francisco, Toronto, London, (1964).

[8] Halanay A. and Rasvan V. Applications of Liapunov Methods in Stability, Kluwer Academic Publishers, Dordrecht, Boston, London, (1993).

[9] Hirsch M.W. Smale S. Devaney R.L. Differential equations dynamical systems, and an introduction to chaos, Academic Press, (2004).

[10] Migdalovici M., Sireteanu T, and Videa E.M., (2010), Control of Vibration of Transmission Lines, International Journal of Acoustics and Vibration, 15(2), pp. 65-71.

[11] Migdalovici M. Vladareanu L. Baran D. Vladeanu G. Radulescu M., (2015), Stability Analysis of Walking Robot Motion, Procedia Computer Science 65:233-240 doi:10.1016/j.procs.2015.09.117.

[12] Parlet B.N. Global convergence of the basic QR algorithm on Hessenberg matrices, Math. of Computation, 22, pp: 803-817, (1968), DOI: 10.2307/2004579.

[13] Pearl J. Causality: Models, Reasoning, and Inferences, Cambridge University Press. (2009).

[14] Pop N. Vladareanu L. Popescu I.N. Ghiţă C. Gal I.A. Cang S. Yu H. Bratu V. Deng M. "A numerical dynamic behaviour model for 3D contact problems with friction", Computational Materials Science, Volume 94, November 2014, pp. 285-291, (2014).

[15] Staretu I. and Jitariu S. (2015), "Reconfigurable Anthropomorphic Gripper with Three Fingers: Synthesis, Analysis, and Simulation", Applied Mechanics and Materials, Vol. 762, pp. 75-82.

[16] Vladareanu L. Io I. Velea L.M. Mitroi D. \& Gal A. "The Real Time Control of Modular Walking Robot Stability", Proceedings of the 8th International Conference on Applications of Electrical Engineering (AEE'09), Houston, USA (pp. 179-186).

[17] Vladareanu V. Munteanu R.I. Mumtaz A. Smarandache F. and Vladareanu L. The optimization of intelligent control interfaces using Versatile Intelligent Portable Robot Platform, Procedia Computer Science 65: 225 - 232 doi:10.1016/j.procs.2015.09.115.

[18] Vladareanu V. Tont G. Vladareanu L. Smarandache F. The Navigation of Mobile Robots in NonStationary and Non-Structured Environments, Int. J. Advanced Mechatronic Sys., 5, 4, 2013, 232 243.

[19] Voinea R. Stroe I. Introduction in the Theory of Dynamical Systems (In Romanian), Romanian Academy Printing House, Bucharest, Romania, (2000).

[20] Wilkinson J. H. Convergence of the LR, QR and related algorithms, Computer J. 8, 77-84, (1965).

[21] Wang Hongbo, Dong Zhang, Hao Lu, Feng Yongfei, Xu Peng, Mihai Razvan Viorel, Vladareanu Luige, (2015), Active Training Research of a Lower Limb Rehabilitation Robot Based on Constrained Trajectory, Proceedings of the 2015 Int. Conf. on Advanced Mechatronic Systems, Beijing, China, August, 24-29, https://ieeexplore.ieee.org/document/7287123. 